\title{
Autism Spectrum Disorder and Co-morbidities: A Closer Look at Pathogenesis and Clinical Implications
}

\author{
Xue-Jun Kong* \\ Department of Medicine and Psychiatry, Beth Israel Deaconess Medical Center, USA \\ *Corresponding author: Xue-Jun Kong, Department of Medicine and Psychiatry, Beth Israel Deaconess Medical Center, USA. \\ To Cite This Article: Xue-Jun Kong, Autism Spectrum Disorder and Co-morbidities: A Closer Look at Pathogenesis and Clinical Implications. 2020 \\ - 7(4). AJBSR.MS.ID.001168. DOI: 10.34297/AJBSR.2020.07.001168.
}

Received: 眥 February 18, 2020; Published: 制 February 20, 2020

\begin{abstract}
The overlap in clinical manifestation of Autism Spectrum Disorder (ASD) and its psychiatric co-morbidities, including Schizophrenia (SCZ), are frequently overlooked. In this review, we enumerated the genetic markers, signs of inflammation and gut-microbiome-brain signatures, and excitation/inhibition imbalances shared by ASD and SCZ. We also summarized advancements in early detection as a result of the predictive value of certain biomarkers and in the development of evidence-based emerging treatments for ASD and co-morbidities.
\end{abstract}

Keywords: Co-Morbidities; Genes; Epigenetics; Pathogenesis; Clinical Implications; Early Diagnosis; Emerging Treatments

Abbreviations: ASD: Autism Spectrum Disorder; SCZ: Schizophrenia; CDC: Centers for Disease Control and Prevention; CNVs: Copy Number Variations; MIA: Maternal Immune Activation; BBB: Blood-Brain Barrier; IVIG: Intravenous Immunoglobulin

\section{Introduction}

Autism Spectrum Disorder (ASD) is a group of neurodevelopmental conditions characterized by social deficit and repetitive behaviors. The Centers for Disease Control and Prevention (CDC) declared ASD an urgent public health concern not only due to its rapid rise in prevalence but also its high rate of co-morbidities. It has been reported that $95 \%$ of ASD individuals have one or more co-morbidities [1-4]. In addition to medical and somatic complications, ASD has symptoms in common with many psychiatric disorders. Particularly, ASD and Schizophrenia (SCZ) have substantial symptomatic overlap related to impaired social communication, abstract reasoning, and working memory [1-4]. This mini review will take a closer look at the biological underpinnings of this shared fundamental pathogenesis and at the future implications of research into early diagnosis and treatments.

\section{Common Genetic Markers of ASD and its Co-Morbidities}

A study that analyzed the genes of twins with ASD concluded that the susceptibility to ASD has both significant genetic heritability and substantial environmental influences [5]. Interestingly, many genetic overlaps of ASD with SCZ and other neuropsychiatric disorders were identified. Carroll \& Owen [6] recognized that the specific deletions associated with both ASD and SCZ at 22q11.2, 1q21.1, and 15q13.3. Most notably, 22q11.2 is a critical region for both disorders [7-9] demonstrated that SNPs in the 3'-UTR of NRXN1, a gene involved in inhibitory and excitatory synaptic differentiation, significantly decreases white matter volumes in whole brain and frontal lobe, while impairing sensorimotor performance; interestingly, NRXN1 is a risk gene for both ASD and SCZ [9]. Copy number variations (CNVs) in the gene CYFIP1 [10], resulting in altered CYFIP1 expression and changes in synaptic inhibition and excitation, were also found to be associated with ASD, SCZ and intellectual disability. These genetic overlaps and clusters explain their shared pathogenesis and corresponding core symptoms.

\section{Inflammation and Gut-Microbiome Bridge Between ASD and Co-Morbidities}

Many inflammatory factors interfere with genetic expression and penetrations. Prata et al. [11] illustrated the inflammatory mechanism bridging ASD and SCZ: elevated circulation of cytokines, such as IL-6, TNF $\alpha$, IL-1 $\beta$, and INFY, increased levels of oxidative 
stress and expression of microRNAs are present in both ASD and SCZ [11]. While these atypical signs do disrupt cellular processes throughout the immune system and brain, they may prove useful as potential biomarkers for ASD and its co-morbidities. The maternal immune activation (MIA) model indicated a link between maternal infection and neurodevelopmental brain disorders, including earlyonset ASD and late-onset SCZ. In both of these disorders, abnormal methylation of the MECP2 gene results in aberrant epigenetic control of MeCP2 protein expression and dysregulated GABAergic signaling in different brain regions [12-15], overviewed the vulnerability-stress-inflammation model of SCZ.

This model posits that the "first hit" to neurodevelopment is maternal infection directly interfering with CNS development, and the "second hit," at a later stage, is stress exposure, triggered inflammation cascade and symptom onset [15]. The presentation of ASD and/or SCZ may be related to the timing and degree of the first and second hits. Anti-NMDA receptor encephalitis usually presents in young adults with new onset of psychosis [16] seizure and cognitive changes but was also reported in preschoolers who presented with new onset of ASD like symptoms [17]. Autoimmunity pathology found in both SCZ [18-20] and ASD [21] have many similarities, yet certain differences in autoantibody profiles indicate differences in protein targets and relevant brain regions. Microbiome imbalance could be the key determinant of inflammation in ASD, SCZ [22-26] and other psychiatric disorders $[24,25]$. Immune activation triggers GI inflammation and produces pro-inflammatory cytokines in the blood, and, when crossing the blood-brain barrier (BBB), cytokines activate microglial cells and astrocytes, resulting in CNS inflammation. Gut-brain axis signaling has been widely reported and implicated in neuro-psychiatric disorder manifestation [13,22-26]. Gut microbiome studies have opened a new avenue for understanding the common inflammatory mechanisms of ASD and its co-morbidities.

\section{Early Diagnosis and Recognition of ASD and Co- Morbidities}

These biological findings have provided promising biomarkers for early recognition and diagnosis. Early diagnosis and early intervention impact the prognosis of these conditions significantly. For ASD, standardized tests, such as ADOS, are largely subjective and not reliable until age 2 . Even then, these tests are frequently not readily available and miss the early clinical signs, which further delays diagnosis. Similarly, ASD co-morbidities are also frequently overlooked during diagnosis and differential diagnosis due to the atypical presentations and overlapped symptoms. Research into objective biomarkers has attracted a lot of attention. Prenatal and neonatal inflammatory markers [27] could hold some predictive value. Elevation of cytokines indicate that inflammation may be an important link between ASD and SCZ [11].
However, there is nota validated panel that can provide a reliable inflammatory signature for these distinct disorders. Preliminary data indicates that gut and oral microbiome signatures [28] may have predictive value for the diagnosis of ASD and ASD subtypes. Recent research offers the first evidence that patients with SCZ show marked disturbances of gut bacterial taxa composition with a decreased microbiome diversity index which are associated with specific SCZ phenotypes, symptom severity, and treatment response [29]. Only overlapping partially, SCZ microbiome disturbances are distinct from those found in ASD. Further studies are warranted to identify microbiome characteristics unique to different psychiatric disorders for the purpose of reliable early detection and accurate differential diagnosis. Burgeoning biotechnologies also offer differential diagnosis value. Eye tracking with a developmental milestone-appropriated paradigm [30] has been reported as a potential early marker for ASD [31,32]. highlighted both shared and distinct social cognitive processes in SCZ and ASD that may contribute to their characteristic social disabilities [32].

Moreover Hazlett HC et al. [33] reported MRI brain surface change at 6-12 months of age and Emerson et al. [34] illustrated functional connectivity change at 6 months of age; both longitudinal studies demonstrated high predictive validity for ASD diagnosis at 24 months. Similarly, an EEG study found that increased cortical reactivity to repeated tones at 8 months correlated with later ASD development [35]. Using electrophysiology and fMRI on both SCZ and ASD participants, researches showed equivalent deficits in facial emotion recognition and motion sensitivity but markedly different profiles of physiological dysfunction. The specific pattern of deficits observed in each group may help guide the development of treatments designed to down-regulate versus up-regulate visual processing within the respective clinical groups [36,37] used the latest advances in deep learning to formulate a personalized machine learning framework for characterizing the ASD children's facial expression, head and body movement, hand gesture, voice, and blood pressure during robot-assisted autism therapy. This study collected a multimodal dataset of 35 ASD children from different countries and confirmed a high agreement between the perception of the robot and the clinical experts in the estimation of effect and engagement of children with ASD (interclass correlation of $60 \%$ ). Studies on the similarities and differences among ASD, SCZ, and other psychiatric disorders via digital tools and artificial intelligence will provide significant diagnostic value in the future.

\section{Emerging Treatments for ASD and Co-Morbidities}

Traditionally ASD treatment has been largely behaviorally based, and SCZ treatments have been focused on symptomatic relief. With the understanding of the shared pathophysiology mentioned above, evidence-based treatments are under preclinical and clinical trial testing and many have demonstrated initial efficacy. To treat the inflammatory and autoimmunity abnormalities in 
ASD [38,39] demonstrated intravenous immunoglobulin (IVIG) treatment has significant efficacy in ASD individuals [40] reported symptom improvement with Rituximab for pediatric autoimmune and inflammatory CNS disease. Sulforaphane, a well-known antiinflammatory and the antioxidant natural product, was reported to have beneficial effects for both ASD [41] and SCZ [42].

Cannabidiol (CBD) oil has been experimentally used in ASD with some improvement in ASD comorbid symptoms [43]. To change the microbiome ecosystem, the treatment trials of probiotics [44] and fecal transplant therapy [45] have been reported considerable improvement of core symptoms in ASD, SCZ and other neuropsychiatric conditions [46]. As discussed above, the excitationinhibition imbalance is a key mechanism for ASD and co-morbid neuro-psychiatric disorders. The emerging drugs targeting this pathway, including some off-label, previously approved drugs, have been through extensive preclinical and clinical trials. Arblafen, a GABA-b receptor agonist, showed improved CGI and social adoption skills [47]. Bumetanide, known to modify intracellular chloride concentration and thereby enhancing GABA mediated inhibition, showed improved CARS, SRS and CGI [48]. These emerging therapies could be beneficial for ASD and other psychiatric comorbidities with shared pathogenesis.

\section{Conclusion}

The ASD epidemic is a medical and public health crisis that is exacerbated by the high incidence of psychiatric co-morbidities. ASD and SCZ, in addition to other neuro-psychiatric disorders, overlap at multiple levels, including genetics, epigenetics, and pathogenesis. Early recognition and diagnosis of ASD and its co-morbidities significantly impact the outcome and prognosis. Evidence-based target therapies on the horizon for ASD, SCZ, and other neuropsychiatric disorders, while promising, require further investigation. Research identifying signature biomarkers will facilitate both diagnosis and differential diagnosis of each distinct condition, which will provide more precise treatment targets and will lead the way for the development of new therapeutic modalities that curb the core symptoms and disease mechanisms.

\section{Acknowledgements}

I appreciate reviews and comments for this manuscript from Drs. Cunjian Dong, Yiqing Song and Haibin Wang. I appreciate Dr. Xiaochun Wang and Mr. Kevin Liu for their assistance in formatting and searching references. I appreciate Mr. Cullen D. Clairmont for his English proofing.

\section{Conflict of Interest}

None

\section{References}

1. Gillberg C, Fernell E (2014) Autism Plus Versus Autism Pure. J Autism Dev Disord 44(12): 3274-3276.
2. Romero M, Aguilar JM, Del-Rey-Mejías Á, Mayoral F, Rapado M, et al. (2016) Psychiatric comorbidities in autism spectrum disorder: A comparative study between DSM-IV-TR and DSM-5 diagnosis. Int J Clin Health Psychol 16(3): 266-275.

3. Houghton R, Ong RC, Bolognani F (2017) Psychiatric comorbidities and use of psychotropic medications in people with autism spectrum disorder in the United States. Autism Res 10(12): 2037-2047.

4. Schendel DE, Overgaard M, Christensen J, Hjort L, Jørgensen M, et al. (2016) Association of Psychiatric and Neurologic Comorbidity With Mortality Among Persons With Autism Spectrum Disorder in a Danish Population. JAMA Pediatr 170(3): 243-250.

5. Hallmayer J, Cleveland S, Torres A, Phillips J, Cohen B, et al. (2011) Genetic heritability and shared environmental factors among twin pairs with autism. Arch Gen Psychiatry 68(11): 1095-1102.

6. Carroll LS, Owen MJ (2009) Genetic overlap between autism, schizophrenia and bipolar disorder. Genome Med 1(10): 102.

7. Merico D, Zarrei M, Costain G, Ogura L, Alipanahi B, et al. (2015) WholeGenome Sequencing Suggests Schizophrenia Risk Mechanisms in Humans with 22q11.2 Deletion Syndrome 5(11): 2453-2461.

8. Clements CC, Wenger TL, Zoltowski AR, Bertollo JR, Miller JS, et al. (2017) Critical region within 22q11.2 linked to higher rate of autism spectrum disorder. Mol Autism 8: 58.

9. Voineskos AN, Lett TA, Lerch JP, Tiwari AK, Ameis SH, et al. (2011) Neurexin-1 and frontal lobe white matter: an overlapping intermediate phenotype for schizophrenia and autism spectrum disorders. Plos one 6(6): 20982.

10. Davenport EC, Szulc BR, Drew J, Taylor J, Morgan T, et al. (2019) Autism and Schizophrenia-Associated CYFIP1 Regulates the Balance of Synaptic Excitation and Inhibition. Cell Rep 26(8): 2037-2051.

11. Prata J, Santos SG, Almeida MI, Coelho R, Barbosa MA (2017) Bridging Autism Spectrum Disorders and Schizophrenia through inflammation and biomarkers-pre-clinical and clinical investigations. J Neuroinflammation 14(1): 179

12. Bergdolt L, Dunaevsky A (2019) Brain changes in a maternal immune activation model of neurodevelopmental brain disorders. Prog Neurobiol 175: 1-19.

13. Conway F, Brown A (2019) Maternal Immune Activation and Related Factors in the Risk of Offspring Psychiatric Disorders. Front Psychiatry 10: 430 .

14. Basil P, Li Q Dempster EL, Mill J, Sham PC, et al. (2014) Prenatal maternal immune activation causes epigenetic differences in adolescent mouse brain. Transl Psychiatry 4(9): 434.

15. Müller N, Bechter K (2013) The mild encephalitis concept for psychiatric disorders revisited in the light of current psychoneuroimmunological findings. Neurol Psychiatry Brain Res 19(3): 87-101.

16. Giné Servén E, Boix Quintana E, Guanyabens Buscà N, Casado Ruiz V, Torres Rivas C, et al. (2019) Considerations of psychotic symptomatology in anti-NMDA encephalitis: Similarity to cycloid psychosis. Clin Case Rep $7(12):$ 2456-2461.

17. Hacohen Y, Wright S, Gadian J, Vincent A, Lim M, et al. (2016) N-methyld-aspartate (NMDA) receptor antibodies encephalitis mimicking an autistic regression. Dev Med Child Neurol 58(10): 1092-1094.

18. Marinas JE, Matveychuk D, Dursun SM, Baker GB (2019) Neuroimmunological antibody-mediated encephalitis and implications for diagnosis and therapy in neuropsychiatry. Acta Neuropsychiatr 3: $1-9$.

19. Severance EG, Dickerson FB, Yolken RH (2018) Autoimmune phenotypes in schizophrenia reveal novel treatment targets. Pharmacol Ther 189 184-198. 
20. Cremaschi L, Kardell M, Johansson V, Isgren A, Sellgren CM, et al. (2017) Prevalences of autoimmune diseases in schizophrenia, bipolar I and II disorder and controls. Psychiatry Res 258: 9-14.

21. Braunschweig D, Krakowiak P, Duncanson P, Boyce R, Hansen RL, et al. (2013) Autism-specific maternal autoantibodies recognize critical proteins in developing brain. Transl Psychiatry 3(7): 277.

22. Strati F, Cavalieri D, Albanese D, De Felice C, Donati C, et al. (2017) New evidences on the altered gut microbiota in autism spectrum disorders. Microbiome 5(1): 24.

23. Hsiao EY, McBride SW, Hsien S, Sharon G, Hyde ER, et al. (2013) Microbiota modulate behavioral and physiological abnormalities associated with neurodevelopmental disorders. Cell 155(7): 1451-1463.

24. Akhondzadeh S (2019) Microbiome and Schizophrenia. Avicenna J Med Biotechnol 11(4): 269.

25. Vindegaard N, Speyer H, Nordentoft M, Rasmussen S, Benros ME (2020) Gut microbial changes of patients with psychotic and affective disorders: A systematic review. Schizophr Res 0920-9964(19): 30584-30585.

26. Zhu F, Guo R, Wang W, Ju Y, Wang Q et al. (2019) Transplantation of microbiota from drug-free patients with schizophrenia causes schizophrenia-like abnormal behaviors and dysregulated kynurenine metabolism in mice. Mol Psychiatry.

27. Zerbo O, Yoshida C, Grether JK, Van de Water J, Ashwood P, et al. (2014) Neonatal cytokines and chemokines and risk of Autism Spectrum Disorder: the Early Markers for Autism (EMA) study: a case-control study. J Neuroinflammation 11: 113

28. Kong X, Liu J, Cetinbas M, Sadreyev R, Koh M, et al. (2019) New and Preliminary Evidence on Altered Oral and Gut Microbiota in Individuals with Autism Spectrum Disorder (ASD): Implications for ASD Diagnosis and Subtyping Based on Microbial Biomarkers. Nutrients 11(9): 2128.

29. Agorastos A, Bozikas VP (2019) Gut microbiome and adaptive immunity in schizophrenia. Psychiatriki 30(3): 189-192.

30. Wan G, Kong X, Sun B, Yu S, Tu Y, et al. (2019) Applying Eye Tracking to Identify Autism Spectrum Disorder in Children. J Autism Dev Disord 49(1): 209-215.

31. Kong X, Wang B, Park J, Kong J. (2017) Introduction of a New VideoBased Eye Tracking Paradigm for Early Detection of ASD. NAJ Med Sci 10(4): 133-135.

32. Sasson NJ, Pinkham AE, Weittenhiller LP, Faso DJ, Simpson C (2016) Context Effects on Facial Affect Recognition in Schizophrenia and Autism: Behavioral and Eye-Tracking Evidence. Schizophr Bull 42(3): 675-683.

33. Hazlett HC, Gu H, Munsell BC, Kim SH, Styner M, et al. (2017) Early brain development in infants at high risk for autism spectrum disorder. Nature 542(7641): 348-351.

34. Emerson RW, Adams C, Nishino T, Hazlett HC, Wolff JJ, et al. (2017) Functional neuroimaging of high-risk 6-month-old infants predicts a diagnosis of autism at 24 months of age. Sci Transl Med 9(393): 2882.

35. McKinnon CJ, Eggebrecht AT, Todorov A, Wolff JJ, Elison JT, et al. (2019) Restricted and Repetitive Behavior and Brain Functional Connectivity in Infants at Risk for Developing Autism Spectrum Disorder. Biol Psychiatry Cogn Neurosci Neuroimaging 4(1): 50-61.
36. Kolesnik A, Ali JB, Gliga T, Guiraud J, Charman T, et al. (2019) Increased cortical reactivity to repeated tones at 8 months in infants with later ASD. Transl Psychiatry 9(1): 46

37. Martínez A, Tobe R, Dias EC, Ardekani BA, Veenstra VanderWeele J, et al. (2019) Differential Patterns of Visual Sensory Alteration Underlying Face Emotion Recognition Impairment and Motion Perception Deficits in Schizophrenia and Autism Spectrum Disorder. Biol Psychiatry 86(7): 557-567.

38. Rudovic O, Lee J, Dai M, Schuller B, Picard RW (2018) Personalized machine learning for robot perception of affect and engagement in autism therapy. Sci Robot 3(19).

39. Melamed IR, Heffron M, Testori A, Lipe K (2018) A pilot study of highdose intravenous immunoglobulin 5\% for autism: Impact on autism spectrum and markers of neuroinflammation. Autism Res 11(3): 421433.

40. Connery K, Tippett M, Delhey LM, Rose S, Slattery JC, et al. (2018) Intravenous immunoglobulin for the treatment of autoimmune encephalopathy in children with autism. Transl Psychiatry 8(1): 148.

41. Dale RC, Brilot F, Duffy LV, Twilt M, Waldman AT, et al. (2014) Utility and safety of rituximab in pediatric autoimmune and inflammatory CNS disease. Neurology 83(2): 142-150.

42. Singh K, Connors SL, Macklin EA, Smith KD, Fahey JW, et al. (2014) Sulforaphane treatment of autism spectrum disorder (ASD). Proc Natl Acad Sci USA 111(43): 15550-15555.

43. Shiina A, Kanahara N, Sasaki T, Oda Y, Hashimoto T, et al. (2015) An Open Study of Sulforaphane-rich Broccoli Sprout Extract in Patients with Schizophrenia. Clin Psychopharmacol Neurosci 13(1): 62-67.

44. Barchel D, Stolar O, De-Haan T, Ziv-Baran T, Saban N, et al. (2019) Oral Cannabidiol Use in Children with Autism Spectrum Disorder to Treat Related Symptoms and Co-morbidities. Front Pharmacol 9: 1521.

45. Santocchi E, Guiducci L, Fulceri F, Billeci L, Buzzigoli E, et al. (2016) Gut to brain interaction in Autism Spectrum Disorders: a randomized controlled trial on the role of probiotics on clinical, biochemical and neurophysiological parameters. BMC Psychiatry 16: 183.

46. Kang DW, Adams JB, Gregory AC, Borody T, Chittick L, et al. (2017) Microbiota Transfer Therapy alters gut ecosystem and improves gastrointestinal and autism symptoms: an open-label study. Microbiome 5(1): 10.

47. Gorbovskaya I, Kanji S, Liu JC, MacKenzie NE, Agarwal SM, et al. (2019) Investigation of the Gut Microbiome in Patients with Schizophrenia and Clozapine-Induced Weight Gain: Protocol and Clinical Characteristics of First Patient Cohorts. Neuropsychobiology 79(1): 5-12.

48. Veenstra-VanderWeele J, Cook EH, King BH, Zarevics P, Cherubini M, et al. (2017) Arbaclofen in Children and Adolescents with Autism Spectrum Disorder: A Randomized, Controlled, Phase 2 Trial. Neuropsychopharmacology 42(7): 1390-1398.

49. Lemonnier E, Villeneuve N, Sonie S, Serret S, Rosier A, et al. (2017) Effects of bumetanide on neurobehavioral function in children and adolescents with autism spectrum disorders. Transl Psychiatry 7(3): 1056. 